\title{
Using the Genetic Algorithm in Developing a Method for Steganography
}

\author{
Nadia M. Mohammed \\ nadia.m.mohammed@uomosul.edu.iq \\ College of Computer Science and Mathematics \\ University of Mosul, Mosul, Iraq
}

\section{Received on: 20/03/2012}

Accepted on: 28/06/2012

\section{ABSTRACT}

This paper has developed a method for hiding in images, as it was first encrypt the secret message chaoticlly using the chaotic encryption algorithm and secondly execute the steganography in two phases, the first divide the cover image (.BMP, .PNG) to a group of sections (Blocks) with the diagonal sequence and make hiding using the cell of the least Significant Bit (LSB) within (Bytes) of certain randomly, and then using the Genetic Algorithm (GA) and working at the expense of Peak Signal to Noise Ratio(PSNR) for each section after the steganography and then get the best PSNR value of the optimal section (ie, a better distribution of the random sites). The second include a final for all sections (Blocks) depending on the results of the first stage and the best for a random distribution of sites (Bytes) according to the results of genetic algorithm.

Measures such as PSNR, BER, MSE and NC are used to prove the accuracy of the results and efficiency. The application implemented using Matlab 9.

Keywords: Genetic algorithm, LSB, BMP, PNG.

$$
\begin{aligned}
& \text { استخدام الخوارزمية الجينية في تطوير طريقة للإخفاء } \\
& \text { نادية معن محد } \\
& \text { كلية علوم الحاسوب والرياضيات } \\
& \text { جامعة الموصل، الموصل، العراق } \\
& \text { تاريخ قبول البحث: 2012/06/28 } \\
& \text { تاريخ استلام البحث: 2012/03/20 } \\
& \text { الملخص } \\
& \text { اشتمل البحث على تطوير طريقة للإخفاء في الصور ، إذ تم أولا تشفير الرسالة السرية فوضويا باستخدام } \\
& \text { خوارزمية التشفير الفوضوي، وثانيا تطبيق الإخفاء على مرحلتين، الأولى تقسيم الصورة الغطاء (BMP,.PNG.) } \\
& \text { إلى مجموعة من المقاطع (Blocks) ذات تسلسل قطري وإجراء الإخفاء فيها بطريقة الخلية الثنائية الأقل أهمية } \\
& \text { (Bytes) معينة وبشكل عشوائي، ثم باستخدام الخوارزمية الجينية } \\
& \text { Peak Signal to Noise Ratio (PSNR) والتي تعمل على حساب Algorithm Genetic (GA) } \\
& \text { المقاطع بعد الإخفاء وبالتالي الحصـول على أفضل قيمـة لاـ PSNR للمقطع المثالي(أي أفضل توزيع عشوائي }
\end{aligned}
$$

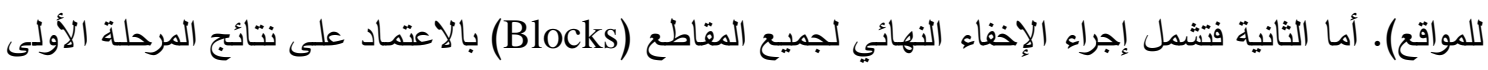

$$
\begin{aligned}
& \text { والخاصة بأفضل توزيع عشوائي للمواقع (Bytes) حسب نتائج الخوارزمية الجينية. }
\end{aligned}
$$


منـذ العهود القديمـة كانـت هنـاك حاجـة ملحسة لإيجـاد وسـائل سرية للحفـاظ على أمنيـة الرسـائل المرسـلة

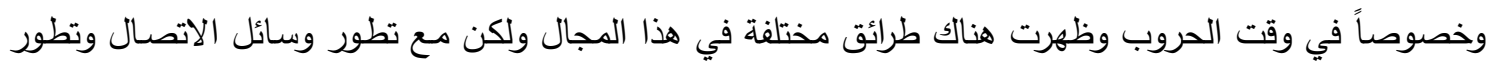

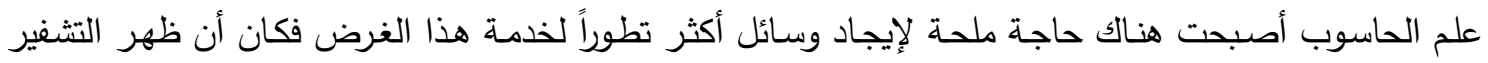
وبالرغم من كونه طريقة جيدة لحفظ المعلومات إلا انه سهل الاكتشاف ويمكن لأي متطفل التلاعب بـه فكانت

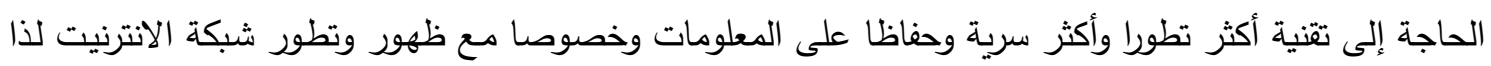

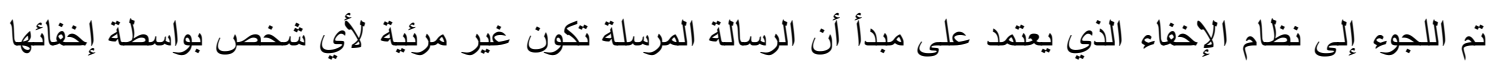
داخل إحدى وسائل الاتصال (الصوت، الصورة والنص، الفيديو) [1]. يمكن تعريف نظام الإخفاء على انه فن وعلم إخفاء المعلومات باستخدام ملف حامل لها (Host) بهدف منع الإنع أي متطفل خارجي من الثك بوجود رسالة مخفية داخل الملف الحامل، وهي وسيلة من وسائل الاتصـال السري بأسلوب يخفي وجود الاتصال.

أن كلمـة Steganography يرجـع أصلها إلى اللغـة اليونانيـة وتتكون مـن المقطعين Steganos وتعني

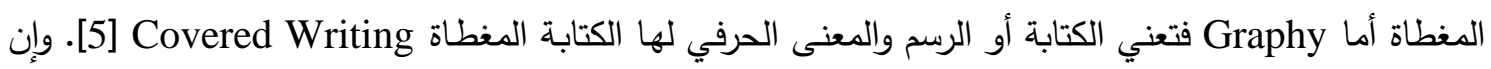

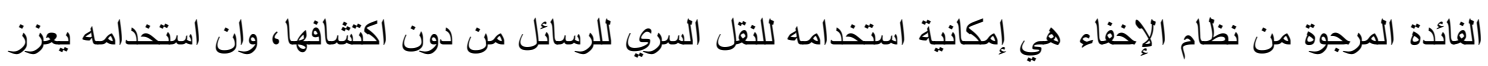
سرية الاتصالات الثخصية ويعد وسيلة مهمة للاتصال خصوصا عبر الانترنيت [7]. تطبق الخوارزمية الجينية بنجاح لإيجاد الحل المقبول ( القريب إلى المثالي) في المسائل المتعلقة بالعلوم

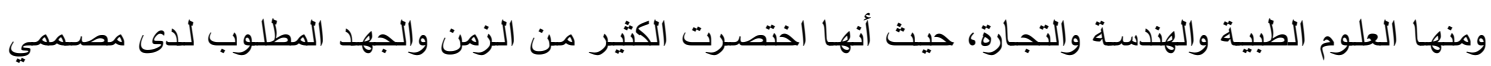
الأنظمة والبرامج، وذلك من خلال إيجادها خوارزمية عامة يعتمد عليها في حل مختلف أنواع المسائل، بدلا من بناء

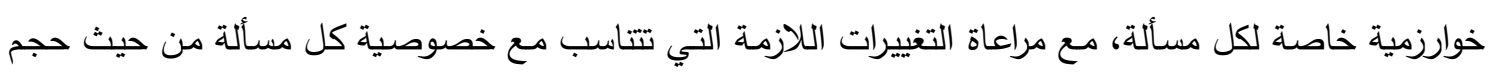
ونوع البيانات المستخدمة وطبيعة دالة الهدف والقيود لكل مسألة [6]. تستخدم الدالة الفوضوية في تثفير البيانات لما تمتاز به من خصائص مثل التعقيد العالي والتصرفات الغير

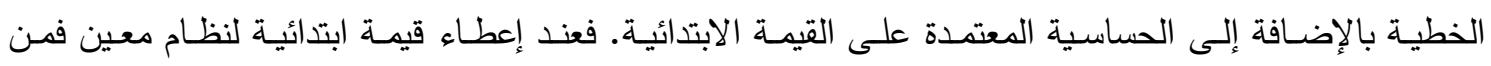

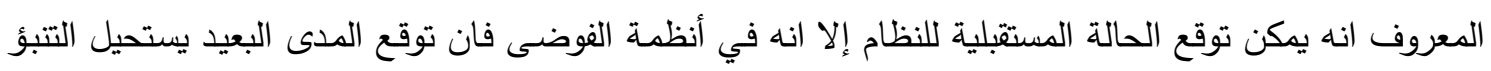

أُّتخدمت الخوارزميـة الجينية (GA) في إخفاء المعلومـات من قبل الباحثين، ففي عام (2010) اقترح

الباحث .El-Zouka,H خوارزمية جينية تعمل على تقليل التشويه الحاصل في البيانات السرية المخفية في الصورة

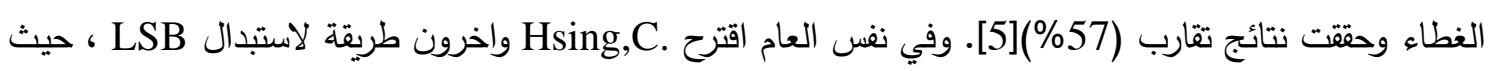

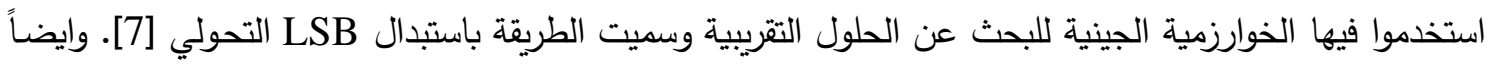
اقترح .Wang, Sh واخرون تقنية إخفاء جديدة بالاعتمـاد على الخوارزمية الجينية، حيث اعتمدت التقنية على بلى

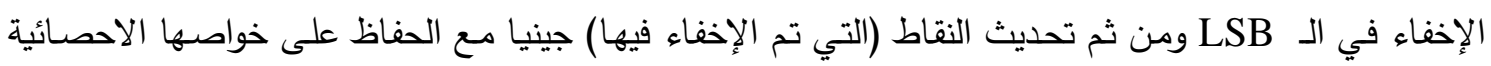
ليصعب اكتشافها [11]. اما في عام (2011) فقدم Mohamed,M وآخرون دراسة لتطبيق تقنية هجينة للا.LSB 
باستخدام مفتاح استبدالي مثالي تم الحصول عليه من خلال الخوارزمية الجينية وذلك لتقليل التثويه الحاصل في

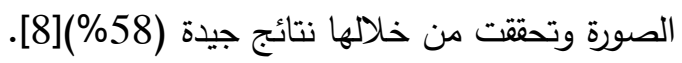

وفي هذا البحث استخدمت الخوارزمية الجينية لتطوير طريقة LSB والحصول على توزيـع عثوائي امثل للإخفاء وكانت النتائج التي الحصول عليها جيدة (68\%).

\section{3فاهيم عامة}

\section{3 الخوارزمية الجينية}

تعرف الخوارزمية الجينية بأنها خوارزمية ذكية يمكن استخدامها لإيجاد حل المسائل المعقدة وتحسينها، كما

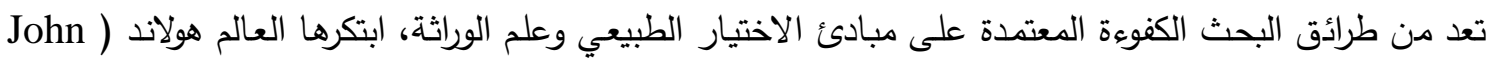

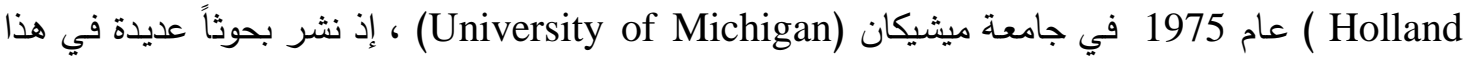

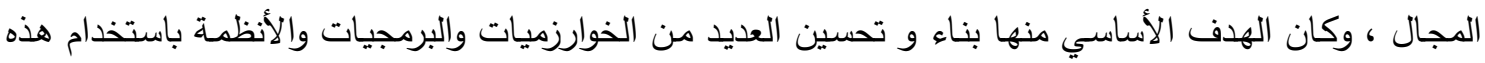

الخوارزمية][8].

يتم حل المسائل المعقدة باستخدام الخوارزميات الجينية بتوليد مجتمع عشوائي يمثل مجموعة الحلول، كل

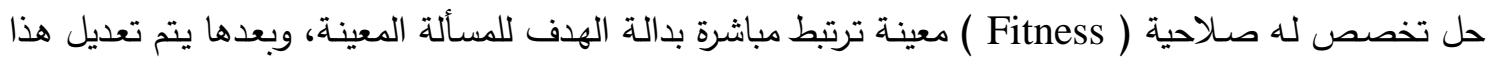

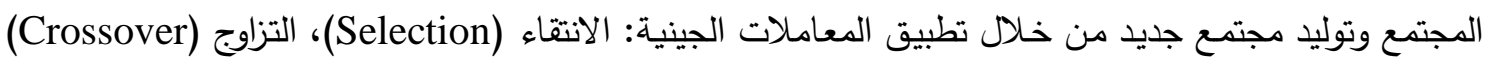

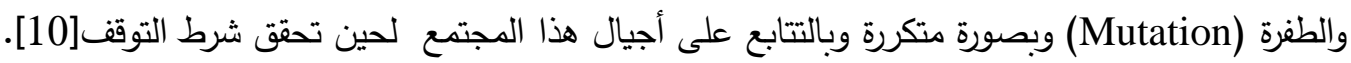

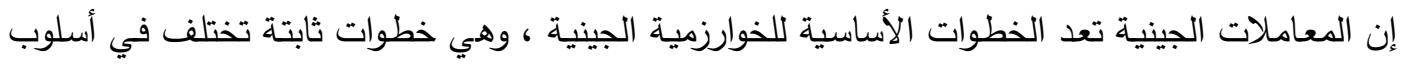

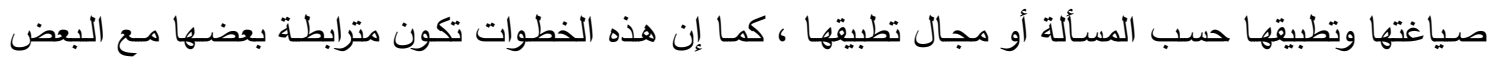
الآخر ، ولا يمكن تطبيق الخوارزمية على أية مسألة ما لم تطبق جميع هذه الخطوات وإلا تفقد الخوارزمية الجينية

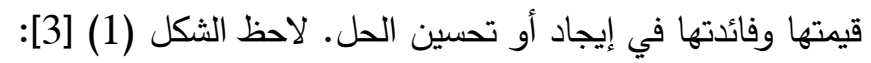

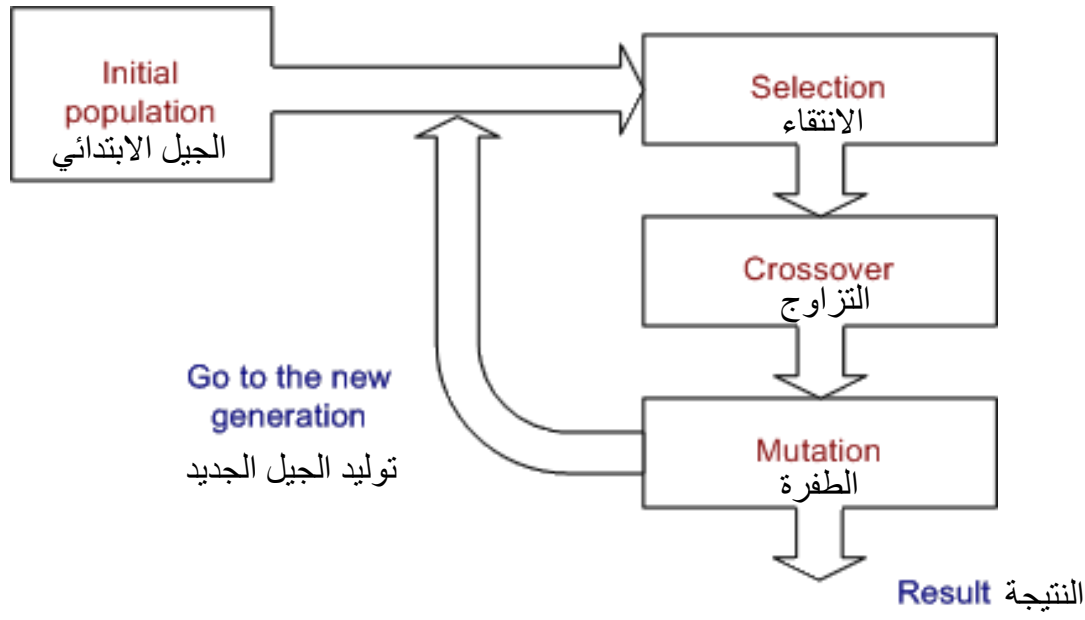

الثكل (1): المخطط العام للخوارزمية الجينية

واحدة من السلوكيات التي تربط الأنظمة غير الخطية والتي تحدث تطورا في القيم المحددة لنظام المعلومات، اذ عدّ اكتشاف هذا النظام العشوائي ثورة ادت الى العديد من القضايا المترابطة ونظريـة الاستقرار وميزات هندسية 
جديدة وعروض لتمييز التواقيع. استخدمت الدالة الفوضوية كاساس لتطوير النماذج الرياضية واجتذبت العديد من الرياضيين بسبب الحساسية العالية للقيمة الابتدائية وتطبيقاتها لمشاكل الحياة اليومية[2].

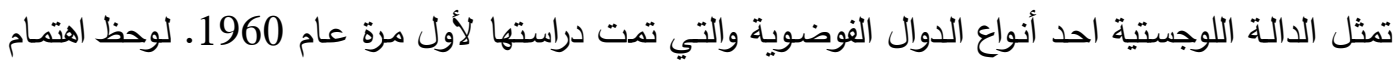

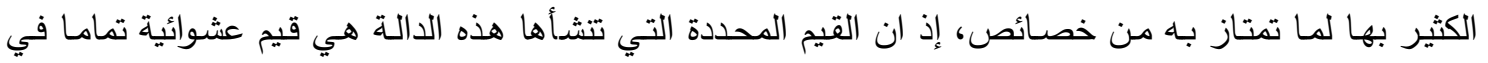
صيغتها (على الرغم من انها تقع ضمن حدود معينة)، وهذه القيم لاتتكرر حتى بعد عدد من الدورات، واهم صفة لهن

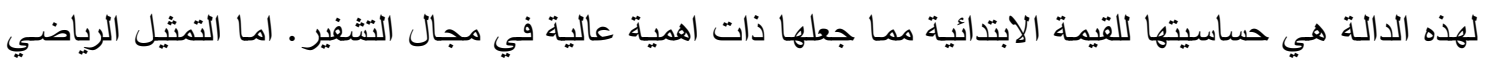
للدالة فهو ممثل بالمعادلة التالية [4]:

$$
X_{n+1}=\mu\left(1-X_{n}\right) X_{n}
$$

$$
\text { حيث ان: }
$$

هي عد+1

(2, (2) (2). لاحظ الثكل (2)

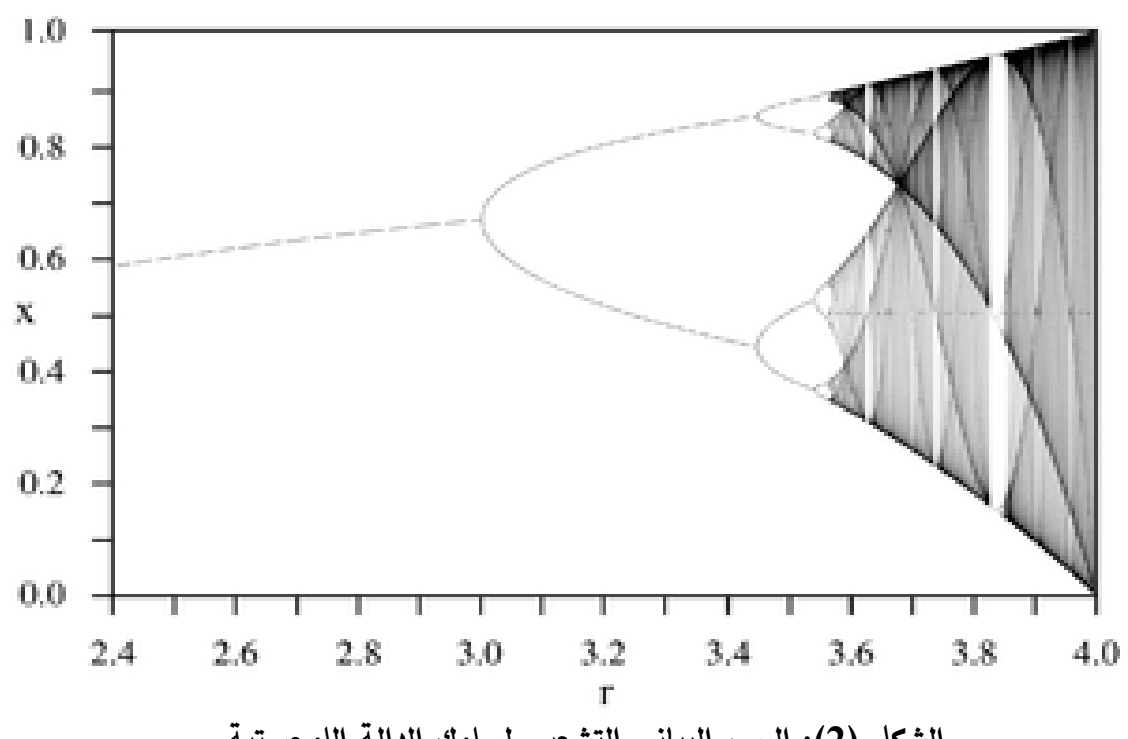

الثكل (2): الرسم البياني التثعبي لسلوك الدالة اللوجستية

3.3

تعد الصور الرقمية من أكثر الوسائط المتعددة استخداما في الكتابة المغطاة بوصفها حاملا للبيانات السرية وذلك بسبب انتثارها الواسع على الإنترنت. ومن أكثر طرائق الإخفاء شيوعا في تضمين البيانات ضمن الصور

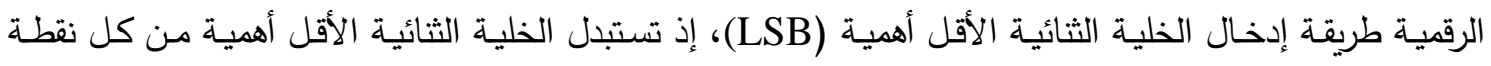
ضوئية في الصور الرقمية بخلية ثنائية من البيانات السرية[1]. يمكن أن تستبدل الخلية الثائية الأقل أهمية الأولى والثانية من النقاط الضوئية مع بقاء الثياء العين البشرية غير

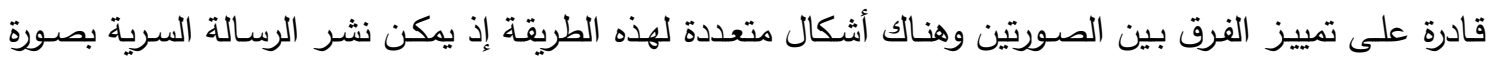

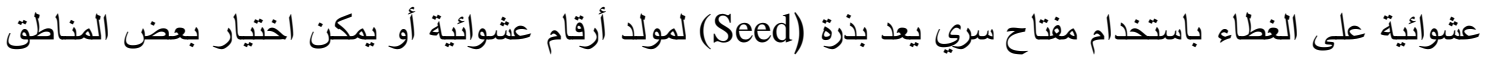

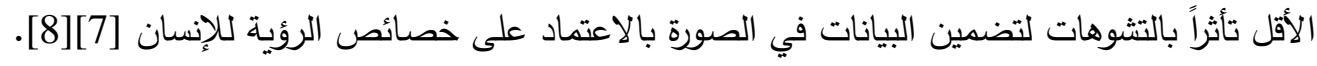
4. خوارزمية التثفير الفوضوي

تستخدم هذه الخوارزمية لتثفير الرسالة السرية (Secret Text) وتتلخص خطواتها بما يأتي[4][9] : 
الخطوة (1): قراءة الرسالة السرية الثنائية ('S).

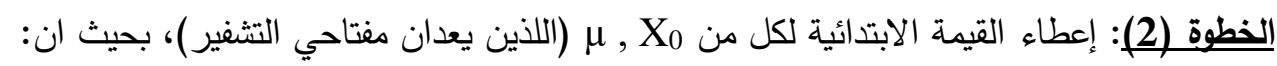

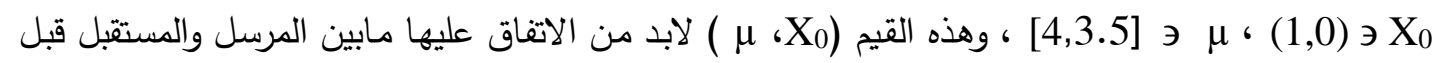

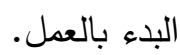

الخطوة (3): توليد سلسلة أرقام حقيقية (R) بحجم (n) وحسب المعادلة (1). الخطوة (4): تحويل السلسلة الناتجة الى سلسلة ارقام ثنائية باستخدام المعادلة الآتية:

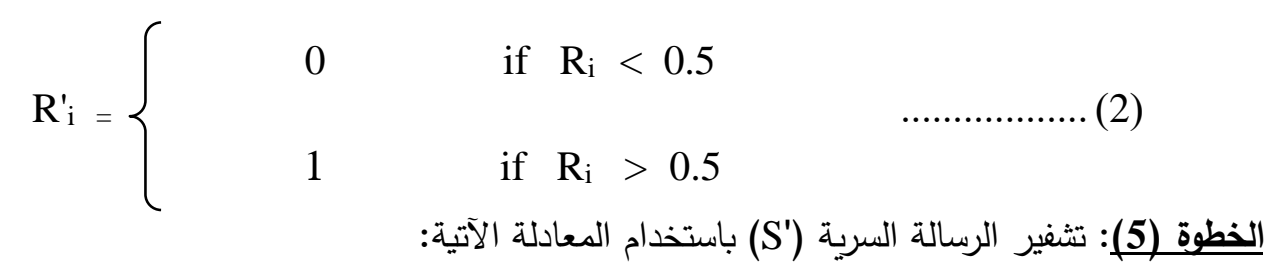

$\mathrm{S}^{\prime \prime}(\mathrm{i})=\mathrm{S}^{\prime}(\mathrm{i}) \oplus \mathrm{R}_{\mathrm{i}}^{\prime}$

$\mathrm{i}=0,1,2, \ldots \ldots \ldots . ., \mathrm{n}$.

$$
\text { حيث ان: }
$$

و الثنكل (3) يوضح عملية تتفير الرسالة السرية.

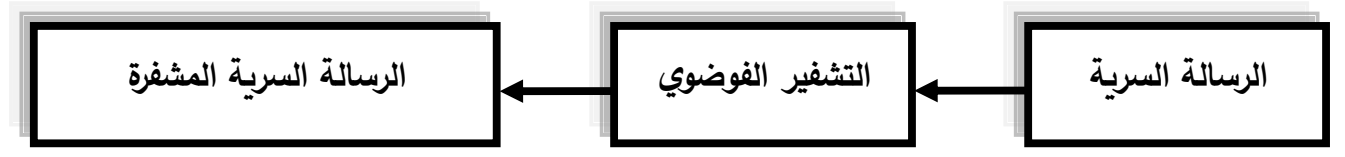

الثكل (3): تثشير الرسالة السرية

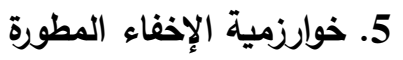

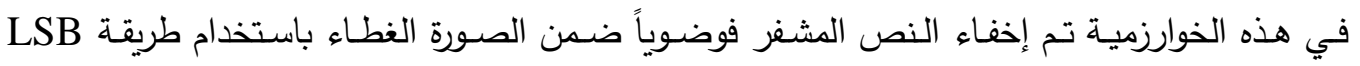

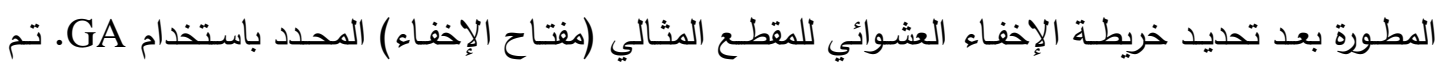

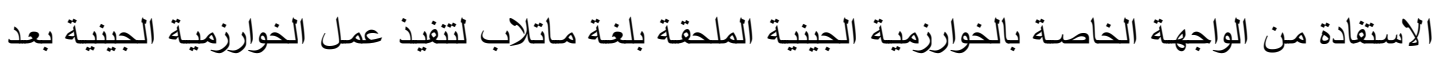

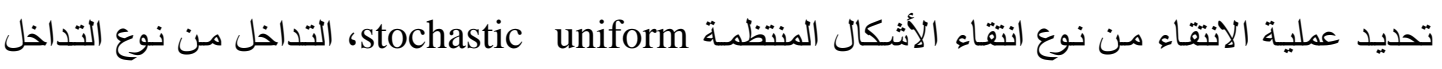
الإبدالي المشت scattered والطفرة من نوع adaptive feasible. وهنا خطوات خوارزمية الإخفاء المطورة:

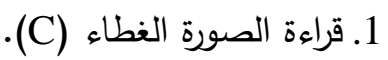

2. قراءة الرسالة السرية (S) (The Prophet Mohammed GBUY is My Teacher). 3. تحويل الرسالة السرية (S) إلى رسالة ثنائية (S'). 4. 2. حساب طول الرسالة السرية الثنائية (n). 5. تشفير الرسالة السرية باستخدام خوارزمية التثفير الفوضوي.

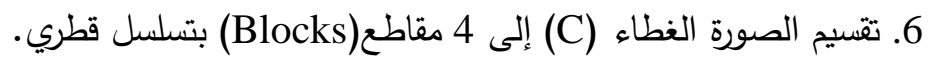
7. تقسيم كل مقطع من المقاطع السابقة إلى 4 مقاطع أخرى بنفس التسلسل القطري. 8. توليد أرقام عشوائية لتحديد مواقع (Bytes) الإخفاء.

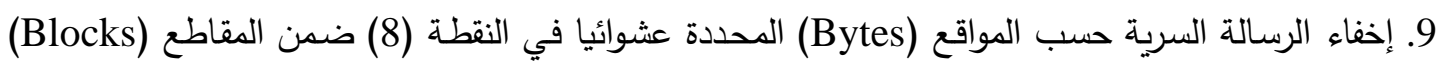

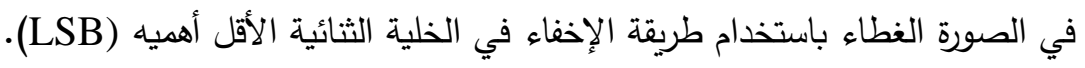
10. تحديد المعاملات المستخدمة في الخوارزمية الجينية وكالاتي: 
a مجتمع ابتدائي من الأفراد، حيث يعد إنشاء الجيل الابتدائي نقطة الانطلاق في حل المسألة، وأن عملية

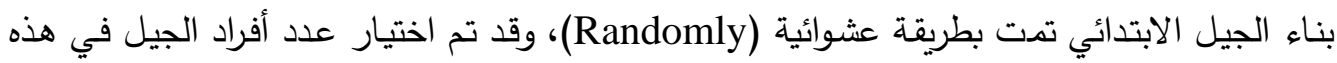

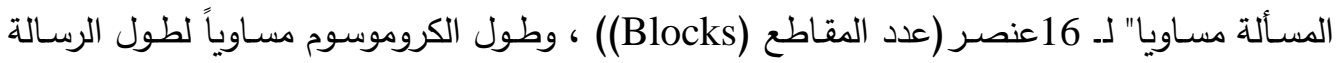

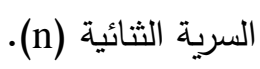

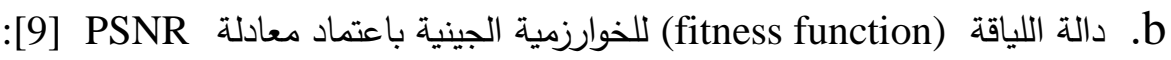
$\mathrm{PSNR}=10 \log _{10}\left[\mathrm{Cmax}{ }^{2} / \mathrm{MSE}\right]$

$$
\mathrm{MSE}=1 /\left(\mathrm{N}^{*} \mathrm{M}\right)^{*} \sum^{\mathrm{N}_{\mathrm{i}=1}} \sum^{\mathrm{M}_{\mathrm{j}=1}}(\mathrm{C}(\mathrm{i}, \mathrm{j})-\mathrm{ST}(\mathrm{i}, \mathrm{j}))^{2}
$$

N,M

الصورة الغطاء قبل وبعد الإخفاء على التوالي. ST، C

$$
\text { Cmax }
$$

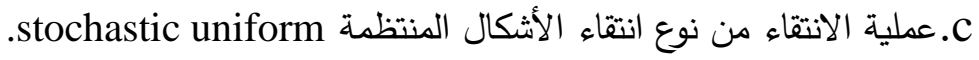

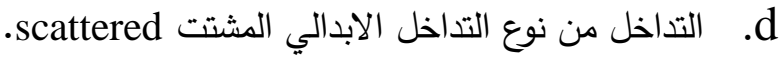

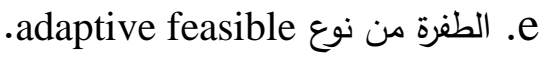

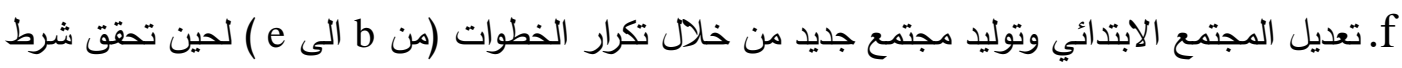
التوقف (الحد الأعلى لعدد الأجيال التي يتم توليدها للحل الأمثل =العدد العدئ 100 (نتيجة التجارب)).

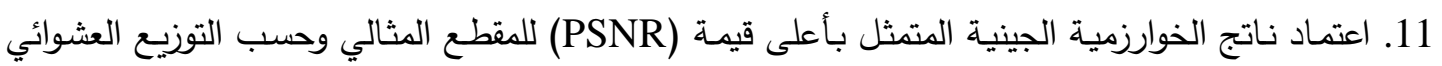

للمواقع (Bytes) المحددة فيه (مفتاح الإخفاء) سيجري الإخفاء بجميع المقاطع عدالمقطع الأخير (16).

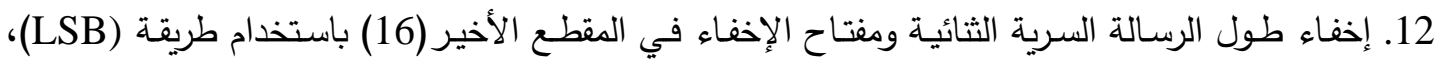

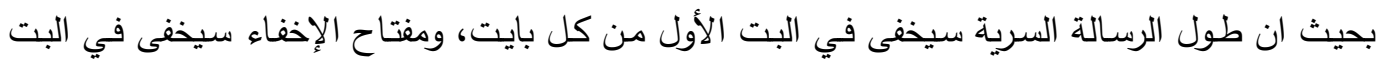
الثاني من كل بايت.

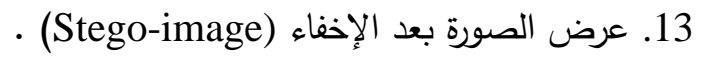
الثكل (4) يوضح خطوات عمل خوارزمية الإخفاء المطورة على صورة رمادية (ROSE.BMP) :

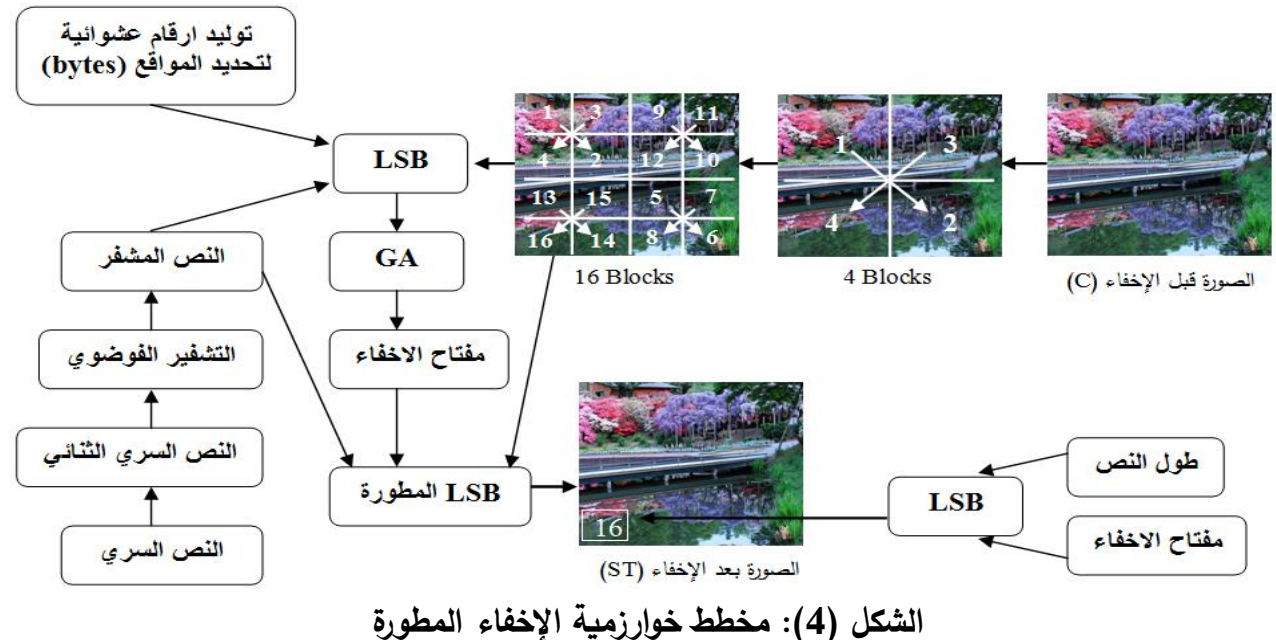




\section{6. خوارزمية الاسترجاع المطورة}

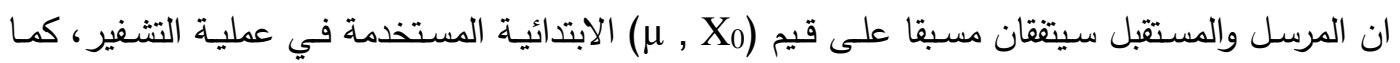

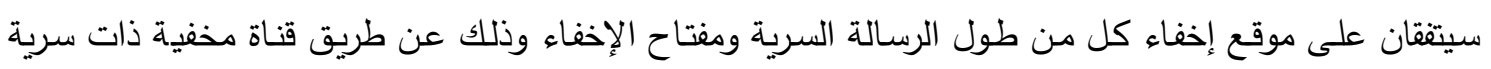
جيدة. وان خوارزمية الاسترجاع ستنفذ كالاتي:

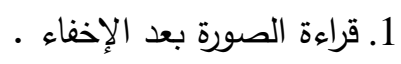

2. تقسيم الصورة الغطاء إلى 4 من المقاطع (Blocks) بتسلسل قطري. 3. تقسيم كل مقطع من المقاطع السابقة إلى 4 مقاطع (Blocks) وبتسلسل قطري ايضا. 4. استرجاع طول الرسالة السرية من المقطع (16) باستخدام طريقة (LSB).

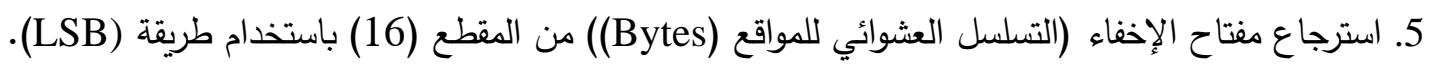

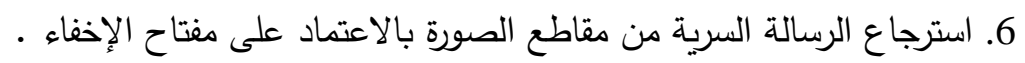

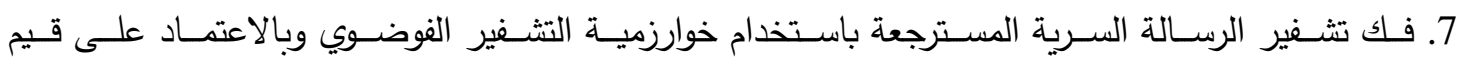

$$
\text { ( المتقق عليها مسبقا. }
$$$$
\text { 8. عرض الرسالة السرية المسترجعة. }
$$

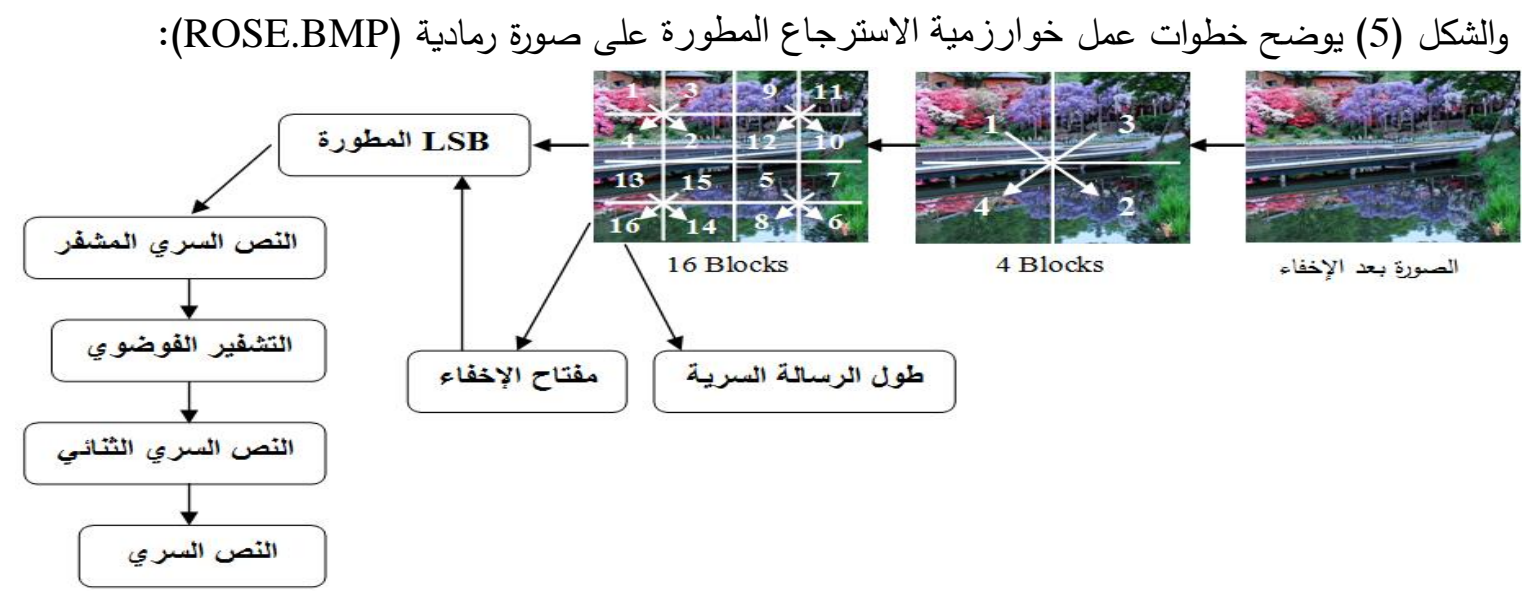

الثكل (5): مخطط خوارزمية الاسترجاع المطورة

7.

لتوضيح الفكرة المطبقة في هذا البحث، تم اعتماد الصورة (SS1.PNG) الموضحة في الثكل(6) كغطاء، When Iam wrong no one forget والنص الآتي بوصفه نصاً سرياً:

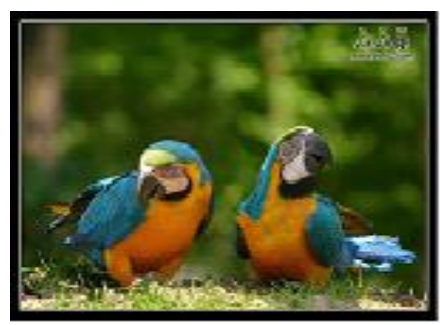

الثكل (6): الصورة الغطاء 
" التشفير الفوضوي : وفيه إستخدمت الدالة اللوجستية بعد تحديد X27=X.927 و = 4 وعدَهما مفتاحا التشفير

للحصول على نص مشفر فوضوياً لاحظ الثكلين (7) و(8).

\begin{tabular}{|llllllll|}
\hline 1 & 1 & 1 & 0 & 1 & 0 & 0 & 0 \\
1 & 0 & 1 & 0 & 0 & 1 & 0 & 0 \\
1 & 0 & 0 & 1 & 0 & 0 & 1 & 0 \\
0 & 1 & 1 & 1 & 0 & 1 & 0 & 1 \\
0 & 1 & 0 & 0 & 1 & 1 & 0 & 0 \\
0 & 0 & 0 & 0 & 0 & 1 & 0 & 1 \\
0 & 0 & 0 & 1 & 1 & 0 & 1 & 1 \\
$\ldots \ldots \ldots \ldots \ldots \ldots \ldots$. & & & & \\
\hline
\end{tabular}

الثكل (8): النص السري المشفر

\begin{tabular}{|cccccccc|}
\hline 0 & 1 & 1 & 1 & 0 & 0 & 1 & 1 \\
0 & 1 & 1 & 1 & 0 & 1 & 0 & 0 \\
0 & 1 & 1 & 0 & 0 & 1 & 0 & 1 \\
0 & 1 & 1 & 0 & 0 & 1 & 1 & 1 \\
0 & 1 & 1 & 0 & 0 & 0 & 0 & 1 \\
0 & 1 & 1 & 0 & 1 & 1 & 1 & 0 \\
0 & 1 & 1 & 0 & 1 & 1 & 1 & 1 \\
$\ldots \ldots \ldots \ldots \ldots \ldots$ & & & & \\
\hline
\end{tabular}

الثكل (7): النص السري الثنائي

• خوارزمية الإخفاء المطورة : وتثمل الآتي:

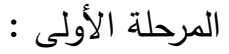

1. تقسيم الصورة الغطاء إلى أربعة مقاطع بتسلسل قطري وإعادة العملية على كل مقطع لينتج 16 مقطعاً.

2. توليد أرقام عشوائية بعدد المواقع في كل مقطع. مثلاً:

.(24،37،23،18،13،34،22،14،15،20،19،16،10،8،11،7،12،3،2،4،1،5،9،6)

3. تطبيق الإخفاء (LSB) لكل المقاطع.

4. تطبيق الخوارزمية الجينية (بالاستفادة من الواجهة الخاصة بالخوارزمية الجينية الملحقة بلغة ماتلاب)

وإيجاد قيم PSNR للدقاطع. لاحظ الجدول(1).

الجدول (1): قيم PSNR لصورة (ROSE.BMP) بعد تطبيق PAP

\begin{tabular}{|c|c|}
\hline PSNR & block \\
\hline 30.8819 & 9 \\
\hline 44.2655 & 10 \\
\hline 42.6103 & 11 \\
\hline $\mathbf{5 5 . 2 1 0 6}$ & 12 \\
\hline 23.4237 & 13 \\
\hline 54.2550 & $\mathbf{1 4}$ \\
\hline 52.8794 & 15 \\
\hline 53.3549 & 16 \\
\hline
\end{tabular}

\begin{tabular}{|c|c|}
\hline PSNR & block \\
\hline 32.5036 & 1 \\
\hline 40.0348 & 2 \\
\hline 50.1719 & 3 \\
\hline 47.6298 & 4 \\
\hline 31.8792 & 5 \\
\hline 25.8971 & 6 \\
\hline 37.7410 & 7 \\
\hline 41.9170 & 8 \\
\hline
\end{tabular}

5. تحديد أعلى قيمة لا PSNR (55.2106). 


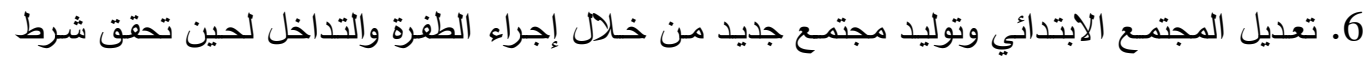

التوقف (الحد الأعلى لعدد الأجيال التي يتم توليدها للوصول للحل الأمثل وهو العدد 100 (نتيجة

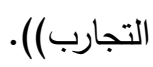

7. الحصول على أعلى PSNR (82.2950) للدقع المثالي(14) (أفضل توزيع عشوائي للإخفاء

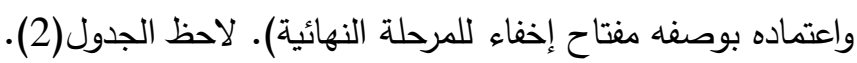

الجدول (2): قيم PSNR النهائية لصورة (ROSE.BMP) بعد تطبيق GAP

\begin{tabular}{|c|c|c|c|c|}
\hline PSNR & block & PSNR & block \\
\hline 40.8585 & 9 & 52.5046 & 1 \\
\hline 47.2895 & 10 & 40.8788 & 2 \\
\hline 42.9103 & 11 & 56.1678 & 3 \\
\hline 77.2106 & 12 & 66.6246 & 4 \\
\hline 43.4236 & 13 & 54.8762 & 5 \\
\hline $\mathbf{8 2 . 2 9 5 0}$ & $\mathbf{1 4}$ & 32.8956 & 6 \\
\hline 70.8704 & 15 & 46.7410 & 7 \\
\hline 74.3543 & 16 & 55.9170 & 8 \\
\hline
\end{tabular}

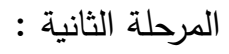

إعتماد ناتج الخطوة السابقة (التوزيع العشوائي للإخفاء) لتتفيذ عملية الإخفاء في مقاطع الصورة.

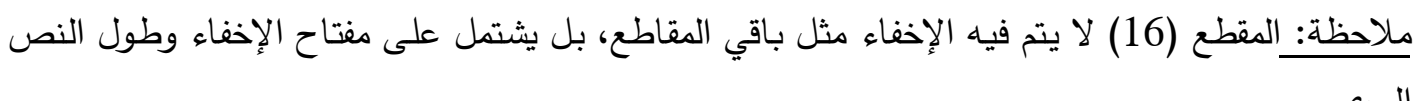

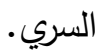

خوارزمية الاسترجاع المطورة : وتثمل الآتي:

1. تقسيم الصورة الغطاء إلى أربعة مقاطع بتسلسل قطري وإعادة العملية على كل مقطع لينتج 16 مقطعاً. 2. استرجاع مفتاح الإخفاء وطول النص السري من المقطع (16).

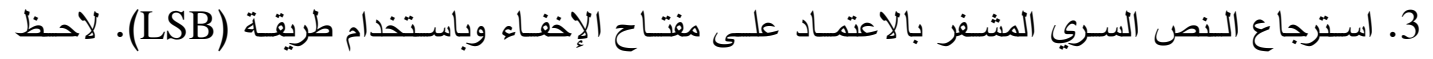

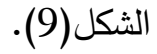

\begin{tabular}{|lllllll|}
\hline 1 & 1 & 1 & 0 & 1 & 0 & 0 \\
0 & 1 & 0 & 1 & 0 & 0 & 1 \\
0 & 0 & 1 & 0 & 0 & 1 & 0 \\
0 & 1 & 0 & 0 & 1 & 1 & 1 \\
0 & 1 & 0 & 1 & 0 & 1 & 0 \\
0 & 1 & 1 & 0 & 0 & 0 & 0 \\
0 & 0 & 0 & 1 & 0 & 1 & 0 \\
0 & - &. &. &. &. &. \\
\hline
\end{tabular}

الثكل (9): النص السري المسترجع (المشفر)

4. فك تثفير النص السري المسترجع باستخدام التشفير الفوضوي بالاعتماد على قيم ( المتفق عليها مسبقاً بين المرسل والمستقبل للحصول على لمبن النص السري: 
When I am wrong no one forget

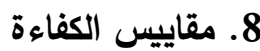

استخدمت المقاييس التالية لقياس كفاءة الطريقة المطورة بالإضـافة إلى مقياس نسبة الضوضـاء بالصورة

PSNR ونسبة الخطأ بالصورة MSE المذكورين سلفا [9]:

Normalize Correlation(NC)

الناتجة بعد الإخفاء وحسب المعادلة الآتية:

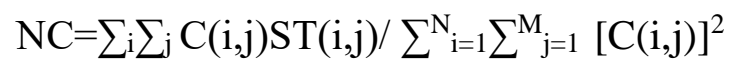

• يستخدم لقياس نسبة الخطأ (عدد الخلايا الخاطئة المسترجعة) وحسب : Bit Error Rate (BER)

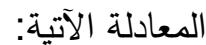

$\mathrm{BER}=(\text { no. of wrong bit/no. of original bit })^{*} 100$

الجدول الأتي يوضح النتائج النهائية بعد تنفيذ الطريقة المطورة على الصورة (ROSE.BMP):

الجدول (4): نتائج تنفيذ الطريقة المطورة على صورة (ROSE.BMP)

\begin{tabular}{|c|c|c|c|c|}
\hline BER & $\mathrm{NC}$ & PSNR & MSE & Block \\
\hline 0 & 0.946 & 42.7036 & 0.029 & 1 \\
\hline 0 & 0.961 & 50.1348 & 0.022 & 2 \\
\hline 0 & 0.981 & 60.8719 & 0.015 & 3 \\
\hline 0 & 0.992 & 67.5298 & 0.011 & 4 \\
\hline 0 & 0.965 & 51.8792 & 0.021 & 5 \\
\hline 0 & 0.975 & 55.8971 & 0.018 & 6 \\
\hline 0 & 0.977 & 57.7810 & 0.017 & 7 \\
\hline 0 & 0.957 & 46.9175 & 0.025 & 8 \\
\hline 0 & 0.949 & 43.8819 & 0.028 & 9 \\
\hline 0 & 0.972 & 54.2605 & 0.019 & 10 \\
\hline 0 & 0.968 & 52.6103 & 0.020 & 11 \\
\hline 0 & 0.987 & 64.2105 & 0.013 & 12 \\
\hline 0 & 0.952 & 45.4233 & 0.026 & 13 \\
\hline 0 & 0.998 & 68.2566 & 0.009 & 14 \\
\hline 0 & 0.984 & 62.6794 & 0.014 & 15 \\
\hline
\end{tabular}


من خلال النتائج الموضحة بالجدول تم الاستتاج إلى أن قيمة BER الناتجة لجميع المقاطع كانت معظمها

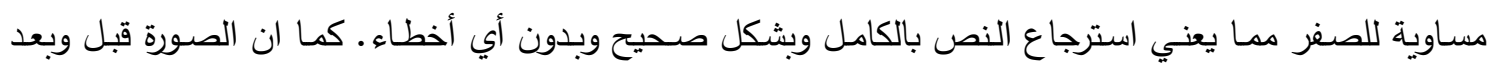

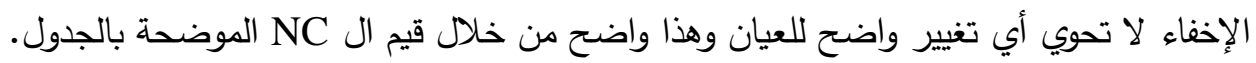

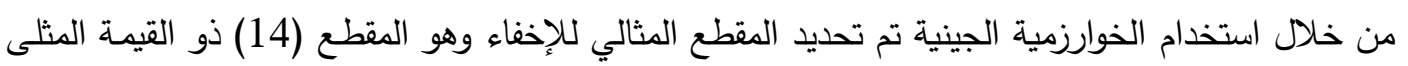
لا PSNR (اكبر قيمة من بين قيمها). أما بالنسبة لقيم MSE الموضحة بالجدول فكانت اغلبها قليلة، ويمكن ملاحظة ان اقل قيمة للا MSE كانت للمقطع (14)(الذي يملك أعلى قيمة PSNR)

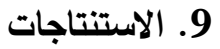

1- أثتتت النتائج قوة وكفاءة الطريقة المطورة. 2- تبين أن تثفير النص باستخدام خوارزمية التثفير الفوضوي يؤدي إلى زيادة سرية الطريقة المطورة وذلك من

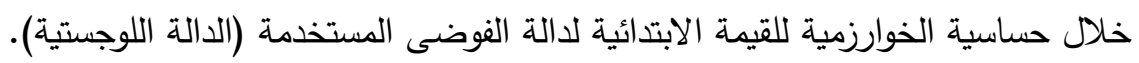

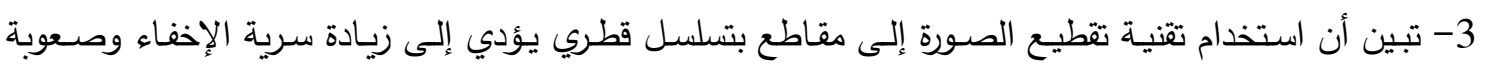
الكثف عن المعلومات المخفية. 4- تم الحصول على قيمة مثلى للا PSNR من خلال استخدام الخوارزمية الجينية ( GA ).

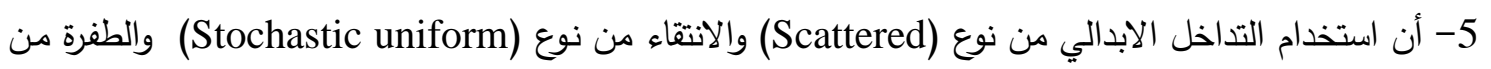
نوع (Adaptive feasible) في الخوارزمية الجينية (GA) يؤدي الى الحصول على أفضل النتائج ( قيمة فئس مثلى للا PSNR) اي (نسبة تثابه صورة الغطاء قبل وبعد الإخفاء). 6- تبين أن استخدام التثفير مع الإخفاء يؤدي إلى زيادة سرية الطريقة المطورة.

1- استخدام طرائق أخرى للتثفير كطريقة DES, RSA.

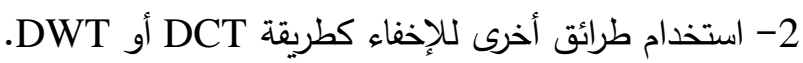
3- استخدام تقنيات ذكائية أخرى في الإخفاء كالثبكات العصبية أو المنطق المضبب. 


$$
\begin{aligned}
& \text { المصادر } \\
& \text { الحمـامي ، عـلاء حسين،(2008) ، "إخفاء المعلومـات الكتابة المخفيـة والعلامـة المائية "، إثراء للنشر }
\end{aligned}
$$

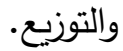

$$
\begin{aligned}
& \text { القدو، سـى جاسـ، سـعيد، ميلاد جـادر، عبد المجيد ، ايـلاف اسـامة، 2010، "التشفير الفوضـوي } \\
& \text { باستخدام مفتاح المقياس الحيوي "، مجلـة الرافدين لعلوم الحاسوب والرياضـيات المجلد (7) العدد(3)، } \\
& \text { الصفحة 186-187. } \\
& \text { بثير ، غصون سالم، 2003، "استخدام الخوارزمية الجينية في مطابقة الصور"، رسالة ماجستير مقدمة إلى } \\
& \text { كلية علوم الحاسوب والرياضيات، جامعة الموصل. }
\end{aligned}
$$

[4] Ahmad, Musheer and Alam, M. Shamsher, 2009, "A New Algorithm of Encryption and Decryption of Images Using Chaotic Mapping", International Journal on Computer Science and Engineering, Vol.2, No.1, page 47.

[5] El-Zouka, H.A, (2010), "Distortion Free Steganography System Based on Genetic Algorithm ", Journal of Information Hiding and Multimedia Signal Prossing. Vol. 1, No. 1, page 11.

[6] Gen, M. , (2000), " Genetic Algorithms and Engineering Optimization ", John Wiley and Sons, Inc.

[7] Hsing,C., Jeng,S., (2010), "Transforming LSB Substitution for Image-based Steganography in Matching Algorithms" , Journal of Information Science and Engineering .

[8] Mohamed, M., Al-Afari, F \& Bamatraf, M., (2011), " Data Hiding by LSB Substitution Using Genetic Optimal Key permutation ", International Arab Journal of e-technology, Vol.2, No.1, pages 11-13.

[9] Mohammad, Shaimaa Sh., (2011), "Encryption and Hiding Water-marking Using A Chaotic Modified Wavelet Transform",Raf. J. of Comp. \& Math's,Vol. 8, No. 2, pages 89-90.

[10] Rutkowski, L.S., (2010), "Artificial Intelligence and Soft Computing" SpringerVerlag Berlin .

[11] Wang, Sh., Yang, B. \& Niu, X., (2010), " A Secure Steganography Method based on Genetic Algorithm ", Journal of Information Hiding and Multimedia Signal Prossing. Vol. 1 , No. 1, page 28. 